\title{
The study on low-quality images Geometric Facial Feature Extraction
}

\author{
Xueping $\mathrm{Liu}^{1,2}$ \\ 1 College of Automation Engineering \\ Nanjing University of Aeronautics and Astronautics \\ Nanjing, China \\ 2 Engineering Training Center \\ Shenyang Aerospace University \\ Shenyang, China \\ liuxueping024@163.com
}

\author{
Yibo Li \\ College of Automation \\ Shenyang Aerospace University \\ Shenyang, China \\ liyibol@sau.edu.cn
}

\begin{abstract}
Face recognition method is a heatedly discussed topic in the field of current pattern recognition and artificial intelligence. Although the face recognition technology has made great achievements, most of the face recognition technologies only focus on the high-resolution images in algorithm design and model training, because the human face images have different sources, some images exhibit very poor resolution, such as fuzzy, high noise and low resolution, which have increased the difficulty of image recognition. Based on the characteristics of low-quality images, this paper first uses wavelet transform to preprocess the images to improve the extraction accuracy of geometric features, adopts the geometric feature normalization method, compares the different selection methods' pros and cons for the face image geometric feature, obtains the corresponding selection method, and effectively improves the recognition effect. This paper describes the experimental analysis of algorithm in the $\mathrm{C}++$ platform. The results show that the algorithm solves the failed recognition of low-quality face images, and improves the face recognition capabilities towards such images.
\end{abstract}

Keywords-Fac Recognition; Low-quality Image; Geometric Feature; Wavelet Transform; Feature Extraction

\section{INTRODUCTION}

Face recognition is one of the most important identity authentication methods based on the biometric identification technology [1]. Besides, the study on face recognition methods has become a heatedly discussed topic in the field of current pattern recognition and artificial intelligence. At present, most of the face recognition technologies only focus on the high-resolution images. By contrast, in respect of the intelligent monitoring, public security suspects' image comparison and other applications, due to different face image sources, some images have low resolution, which has increased the difficulty of image recognition. As a result, how to improve the system's low-quality image recognition ability is also one of the key issues for the face recognition to be solved[2]. However, for low-quality image face recognition, since there is no uniform standard for image resolution, the low-quality image assessment is still a very subjective concept, so various low-quality images can only assess based on its effect on the actual recognition. [3]

Geometric facial feature[4] vectors are usually based on the shape and geometric relationship of face. Brunelli[5] et al proposed some methods, and they used Euclidean distance to characterize the 35-dimensional facial feature vectors, extracted the key points of face organs by using different gray integral projection method, and then obtained the geometric relationships between facial parts through the facial structure. Poggio also worked with Brunelli,they improved the Baron's template matching method and designed an automatic template matching recognition system. Yuille[6]et al proposed different geometric feature extraction mechanism, used the deformable template method to extract the geometric facial features, and also achieved good results.

Face recognition method based on the geometric feature complies with the mechanism of the human face recognition, which is easy to understand[7]. In addition, the geometric intuitive features are relatively stable and less affected by time span[8,9], face pose change, illumination conditions and other factors. Each image only needs to store less feature vectors, so it has less memory and improves the speed of recognition calculation. Therefore, based on the low-quality image facial features, this paper adopts the face recognition method based on geometric features, applies the wavelet transform[10]for image illumination compensation, compares the pros and cons of the different selection methods when selecting the human face geometric features, obtains the corresponding selection results, and improves the effects of low-quality image recognition to some extent.

\section{RELATED WORK}

Typical features of low-quality images include image being too dark or too bright, uneven distribution of gray, indistinct edge contour, not prominent level, noise interference and too low resolution. For the above images, it is impossible to directly obtain useful information from the images for face recognition, hence the image preprocessing. This paper mainly uses the wavelet 
transform for image processing illumination compensation. In the two-dimensional face recognition, non-uniform illumination is often one of the important factors affecting face recognition, and non-uniform face illumination compensation has been a difficult problem in face recognition. Non-uniform illumination compensation achieved in this study combines with the wavelet transform and focuses on the excellent image processing capabilities in the domain. After completion of the face illumination compensation, this paper carries out the face recognition experiment, and proves the illumination compensating effects through face recognition.

The gray image of face $f(x, y)$ is the product of the reflection component $\mathrm{r}(\mathrm{x}, \mathrm{y})$ and illumination component $\mathrm{e}(\mathrm{x}, \mathrm{y})$ :

$$
f(x, y)=r(x, y) \times e(x, y)
$$

Where, $r(x, y)$ stands for the image's fast change, and $\mathrm{e}(\mathrm{x}, \mathrm{y})$ stands for the image's slow change. Take the logarithm for both sides of Formula 2.1, and obtain:

$$
\log [\mathrm{f}(\mathrm{x}, \mathrm{y})]=\log [\mathrm{r}(\mathrm{x}, \mathrm{y})]+\log [\mathrm{e}(\mathrm{x}, \mathrm{y})]
$$

From Formula 2.1 and Formula 2.2, in the spatial domain, the original image's reflection component $r(x, y)$ and illumination component $\mathrm{e}(\mathrm{x}, \mathrm{y})$ has the multiplied relationship. However, in the logarithmic domain, the corresponding part of the reflection component and illumination component $\log [\mathrm{r}(\mathrm{x}, \mathrm{y})]$ and $\log [\mathrm{e}(\mathrm{x}, \mathrm{y})]$ changes into the sum relationship. As a result, in the logarithmic domain, the image illumination compensation is conducted to eliminate the logarithmic domain image's $\log [\mathrm{e}(\mathrm{x}, \mathrm{y})]$ component to the largest degree, which is mainly composed of the low-frequency component in the logarithmic domain. Therefore, in the logarithmic domain, the face image illumination compensation is equivalent to high-pass filtration of the face images. Wavelet transform technology has an excellent high-pass processing capability towards images, so this paper eliminates the low-frequency part in the logarithmic domain by using the two-dimensional wavelet transform multi-level decomposition algorithm and reconstruction algorithm, so as to realize the face image illumination compensation in the logarithmic domain. Specific plans are as follows:

Transform the face image $f(x, y)$ in the spatial domain, and obtain the image in the logarithmic domain $\mathrm{f} 0(\mathrm{x}, \mathrm{y})=$ $\log [\mathrm{f}(\mathrm{x}, \mathrm{y})]$.

Calculate the level-1 two-dimensional discrete wavelet components towards $\mathrm{f} 0(\mathrm{x}, \mathrm{y})$, and get the low-frequency approximate component $\mathrm{A} 1 \mathrm{f} 0(\mathrm{x}, \mathrm{y})$, vertical detail component $D_{1}^{1} \quad \mathrm{f} 0(\mathrm{x}, \mathrm{y})$,horizontal detail component $D_{1}^{2}$ f0(x,y)and diagonal detail component $D_{1}^{3}$ fO(x,y) after level-l wavelet decomposition.

Stop until visual sense can best identify the facial features, otherwise, continue to decompose.

Realize n-level two-dimensional discrete wavelet reconstruction for An f0 $(\mathrm{x}, \mathrm{y})$, so as to get the reconstruction image $\tilde{f} 0(x, y)$ for the logarithmic domain $\mathrm{fO}(\mathrm{x}, \mathrm{y})$.

fo $(x, y)$ minus $f(x, y)$ to get the image element after illumination compensation $\bar{f} 0(x, y)=f 0(x, y)-\tilde{f} 0(x, y)$. "Fig. 1" shows the low-quality image's original image and images after wavelet decomposition:

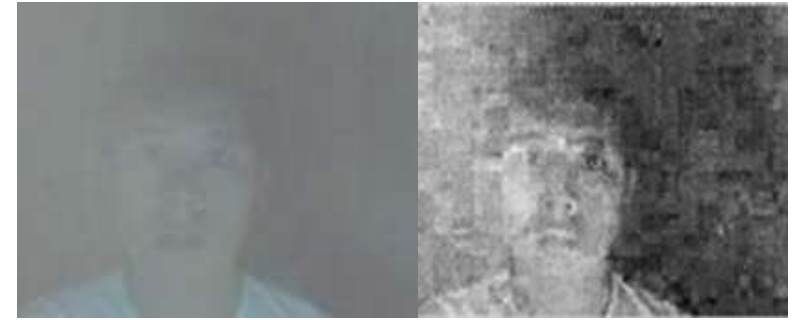

$\begin{array}{ll}\text { (a) Original Image } & \text { (b) Decomposed Image }\end{array}$

Figure 1. Comparison of Decomposed Image Change

In this study, face recognition adopts the method based on the skin color similarity. This paper first takes the face similarity calculation, then conducts the binary calculation, carries out the regional division of face, and finally completes the facial feature mark. The marked eyes, mouths and noses images are shown in "Fig. 2".
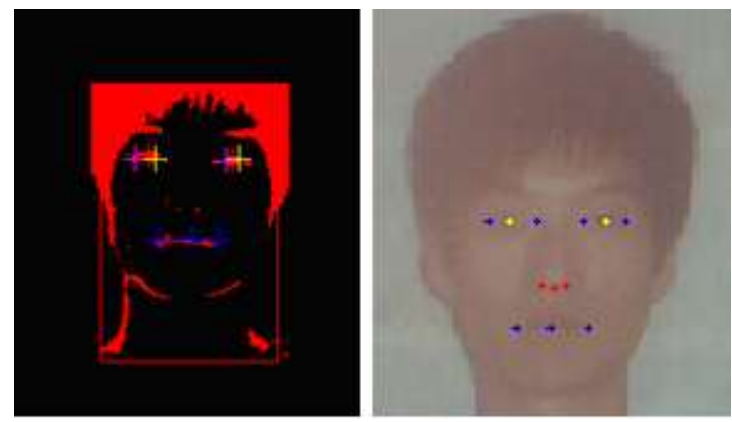

Figure 2. Facial Feature Mark

At the selection stage of facial feature value, this study comprehensively considers the feasibility of feature selection, selects the distance of eyes, width of mouth, the distance from eyes to the center of mouth and other distance parameters as the facial feature test data. Since the size of the face image is uncertain, the extracted distance parameter cannot apply to recognition. The study here adopts the ratio determination method to realize the normalization of the feature values.

The initially selected feature parameters are the ratio relations of two distances, this study specifically takes the distance of eyes, intraocular distance (the distance from the right corner of the left eye to the left corner of right eye), outer eye distance (the distance from the left corner of the left eye to the right corner of right eye), width of mouth and the distance from the center of left eye to the center of mouth. To achieve the normalization of feature values, this study takes the ratio of the first four distances and the last distance as the feature parameter for extraction. In other words, recognition of images with different sizes can be realized by taking the ratio, yet the recognition effect is not ideal. Through research, the denominators of four feature values are the parameter of the distance from the center of left eye to the center of mouth. Therefore, if the position for center of left eye or the center of mouth is not extracted accurately in an image, it will affect the accuracy of all feature parameters. As a consequence, the diversity principle shall be adopted for the selection of denominator, preventing individual parameters incorrectly affecting the overall feature parameters. Considering the angular importance in the geometric location relationship, an angle's cosine is added as the feature parameter. 


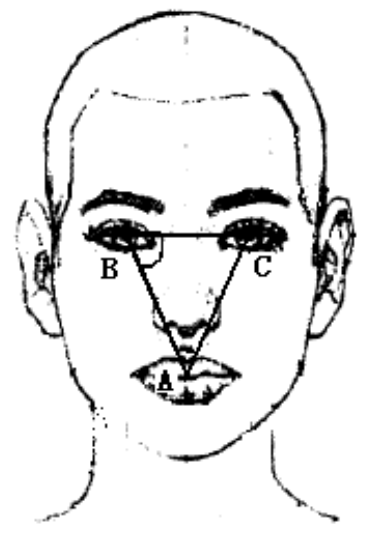

Figure 3. Location Relationship of Geometric Facial Feature

The triangle of the eyes center and mouth center is shown in "Fig. 3" Point A is the center of mouth, whereas Point $\mathrm{B}$ and Point $\mathrm{C}$ are respectively the centers of left eye and right eye. $\angle A B C$ and $\angle A C B$ are two base angles of isosceles triangle with equal size, so this study only takes the cosine value of a base angle $\angle A B C$ and vertex angle $\angle B A C$ as the feature parameter. From the test result of a large number of images, the cosine value of different face image $\angle A B C$ has little difference, which cannot achieve the role of feature parameters. Therefore, this study gives up the parameter of $\angle A B C$ and only retains the cosine feature parameter of $\angle B A C$. Specific implementation is as the following:

$$
\cos \angle B A C=\frac{A B^{2}+A C^{2}-B C^{2}}{2 A B \times A C}
$$

In conclusion, the final Facial feature value is selected to be: the ratio of the distance from the center of eyes as well as the distance from the right corner of left eye to the left corner of mouth; the ratio of the distance from the width of mouth to the center of mouth; the ratio of the distance from the width of mouth and the center of eyes to the center of mouth as well as the cosine value of $\angle B A C$. In such a way, the reasonableness of the required feature parameter selection is implemented.

\section{EXPERIMENTAL RESULTS}

This paper plans the face recognition system based on the geometric feature by using the $\mathrm{C}++$ development platform, adopts the above two plans for extraction of Facial feature value, carries out Facial feature value extraction experiment over a large number of low-quality images, and respectively compares the feature value results obtained from two feature value extraction methods. The below table has listed some feature values' results. Table I shows the results for three people's feature value extraction in the original plan, and Table II shows the results for three people's feature value extraction in the improved plan.
TABLE I. RESUlTS FOR FACIAL FEATURE VALUE EXTRACTION IN THE ORIGINAL PLAN

\begin{tabular}{|c|c|c|c|c|}
\hline $\begin{array}{c}\text { Name of } \\
\text { Sample }\end{array}$ & $\begin{array}{c}\text { First } \\
\text { Feature } \\
\text { Value }\end{array}$ & $\begin{array}{c}\text { Second } \\
\text { Feature } \\
\text { Value }\end{array}$ & $\begin{array}{c}\text { Third } \\
\text { Feature } \\
\text { Value }\end{array}$ & $\begin{array}{c}\text { Fourth } \\
\text { Feature } \\
\text { Value }\end{array}$ \\
\hline Yin & 0.870588 & 0.741176 & 1.07059 & 0.647059 \\
\hline Yin & 0.892473 & 0.741935 & 1.06452 & 0.666667 \\
\hline Zhang & 0.896552 & 0.637931 & 1.10345 & 0.689655 \\
\hline Zhang & 0.862069 & 0.689655 & 1.05172 & 0.672414 \\
\hline Zhou & 0.807692 & 0.501247 & 1.15385 & 0.653846 \\
\hline Zhou & 1.0020135 & 0.689655 & 0.598098 & 0.740741 \\
\hline$\cdots$ & $\ldots$ & $\cdots$ & $\cdots$ & $\cdots$ \\
\hline
\end{tabular}

TABLE II. RESUlTS FOR FACIAL FEATURE VALUE EXTRACTION IN THE IMPROVED PLAN

\begin{tabular}{|c|c|c|c|c|}
\hline $\begin{array}{c}\text { Name of } \\
\text { Sample }\end{array}$ & $\begin{array}{c}\text { First } \\
\text { Feature } \\
\text { Value }\end{array}$ & $\begin{array}{c}\text { Second } \\
\text { Feature } \\
\text { Value }\end{array}$ & $\begin{array}{c}\text { Third } \\
\text { Feature } \\
\text { Value }\end{array}$ & $\begin{array}{c}\text { Fourth } \\
\text { Feature } \\
\text { Value }\end{array}$ \\
\hline Yin & 0.986667 & 0.670732 & 0.743243 & 0.597781 \\
\hline Yin & 0.954023 & 0.666667 & 0.738095 & 0.597475 \\
\hline Zhang & 1.012039 & 0.689655 & 0.754717 & 0.598098 \\
\hline Zhang & 1.041903 & 0.709091 & 0.764706 & 0.609561 \\
\hline Zhou & 0.913043 & 0.693878 & 0.739130 & 0.655612 \\
\hline Zhou & 0.893617 & 0.680000 & 0.723404 & 0.668868 \\
\hline$\cdots$ & $\cdots$ & $\cdots$ & $\cdots$ & $\cdots$ \\
\hline
\end{tabular}

From the above two tables, the improved facial feature value extraction method exhibits better effect, which is reflected that the same sample's same feature value gets very close, yet has distinct features compared with other samples' same feature value. The face recognition is thus realized by respectively applying the minimum distance classifier and geometric classifier. The latter method's feature extraction greatly improves the face recognition rate.

\section{CONCLUSION}

This paper conducts illumination compensation for the low-quality image through the wavelet transform, uses the face detection method based on skin color similarity, realizes the regional division of face through the facial similarity calculation and further binary calculation, and 
finally achieves the mark of face region. After marking the face region, the study adopts the geometric feature extraction method based on knowledge to realize the marking of facial feature and feature value extraction. At last, the study makes use of the minimum distance classifier and geometric classifier to realize the face recognition. Experimental results show that the proposed facial feature extraction has achieved better results than the original plan, playing an important reference role in the geometric feature selection for the future recognition research. In addition, this paper also affirms the effectiveness of low-quality image face recognition technology based on the geometric feature.

\section{REFERENCES}

[1] LI Wu-Jun WANG Chong-Jun ZHANG Wei CHEN Shi-Fu, "A Survey of Face Recognition," Pattern Recognition and Artificial Intelligence, 2006, 1.

[2] Li Xuan, Luo Min, Shi Ronghua, Li li, "Eyes Location Based on Skin Color and Geometry Characteristics in Complex Background," Computer Measurement \& Control, 2005,13 (3).

[3] Chellappa R, Wilson C L, Sirohey S, "Human and machine recognition of faces:a survey[J]," Proc. IEEE,1995,83(5):705-740.
[4] Abate et al.,2007 A.F. Abate,M.Nappi,D.Riccio and G. Sabatino, "2D and 3D face recognition:A survey," Pattern Recognition Letter 28(2007), pp.1885-1906.

[5] Brunelli R,Poggio $T$, "Feature recognition:features versus templates[J]," IEEE Transactions on Pattern Analysis and Machine Intelligence, 1993,15 (10):1042-1052.

[6] Yuille A L,Cohen D,Hallian P, "Facial feature extraction by deformable templates[R]," Technical report 88.2,Harvard Robotics Lab, 1988

[7] Yonghong XU,Jing Hou,Yanru ZHAO, Wenxue HONG, "Quaternion Wavelet Magnitude/Phase and Multi-Scale Local Binary Pattern Representation for Face Recognition.[J]," Journal of Computational Information Systems(JCIS).2011,7(14):5282-5291.

[8] Chun-Nian Fan,Fu-Yan Zhang, "Homomorphic filtering based illumination normalization method for face recognition," Pattern Recognition Letters,2011, 32(10):1468-1479.

[9] Chun-Nian Fan,Hai-Bin Wang,Fu-Yan Zhang, "Improved waveletbased illumination normalization algorithm for face recognition," Proceedings of International Conference on Information Science and Engineering(ICISE 2009),Nanjing,China,, 2009,pp:583-586.

[10] PENG Ye, WANG Shun-fang, DING Hai-yan, "An improved method of face recognition combined with wavelet, Fisher face and geometrical characteristics," Journal of Yunnan University, 2011,33 (S2):215-219. 